\title{
Control of dynamical localization
}

\author{
Jiangbin Gong, ${ }^{*}$ Hans Jakob Wörner, and Paul Brumer \\ Chemical Physics Theory Group, Department of Chemistry, University of Toronto, 80 St. George Street, Toronto, Canada M5S $3 H 6$
}

(Received 25 June 2003; published 3 November 2003)

\begin{abstract}
Control over the quantum dynamics of chaotic kicked rotor systems is demonstrated. Specifically, control over a number of quantum coherent phenomena is achieved by a simple modification of the kicking field. These include the enhancement of the dynamical localization length, the introduction of classical anomalous diffusion assisted control for systems far from the semiclassical regime, and the observation of a variety of strongly nonexponential line shapes for dynamical localization. The results provide excellent examples of controlled quantum dynamics in a system that is classically chaotic and offer opportunities to explore quantum fluctuations and correlations in quantum chaos.
\end{abstract}

DOI: 10.1103/PhysRevE.68.056202

PACS number(s): 05.45.Mt, 32.80.Qk, 05.60.-k

\section{INTRODUCTION}

The quantum kicked rotor (KR) and its classical analog, the standard map, have long served as a paradigm for quantum and classical chaos [1]. Due to its atom optics realization [2], the KR has recently attracted renewed interest. The KR is also of considerable interest in a variety of other fields such as condensed matter physics $[3,4]$, molecular physics $[5,6]$, and quantum information science $[7,8]$.

One well-known quantum effect in a KR is "dynamical localization" (DL) [1]. That is, although a classical kicked rotor displays unrestricted diffusive energy increase due to classical chaos, only a finite number of rotational states are excited in the quantum dynamics, with the quantum excitation probability versus the rotational quantum number typically assuming a characteristic exponential line shape. DL is a pure quantum coherence effect and is therefore very sensitive to decoherence. For example, previous studies have demonstrated that noise [9-11], nonperiodicity in the kicking sequences [12,13], and quantum measurements [7] can destroy DL.

As a coherence effect, DL is also indicative of the possibility of controlling the KR dynamics via quantum effects [14-16]. Indeed, we recently showed that the quantum phases describing the initial rotor quantum state can be manipulated to effectively control quantum fluctuations in quantum chaos and thus enhance or suppress quantum chaotic diffusion $[17,18]$ in a KR. However, manipulating quantum phases in initial states cannot change the unitary evolution operator of the system. Hence, neither the average dynamical localization length nor the characteristic line shape of dynamical localization can be altered in this way.

Motivated by interest in controlled classically chaotic quantum dynamics [17-19], and to gain more insights into the nature of DL, we consider controlling DL and the associated energy absorption via a modified kicked-rotor (MKR) system, in which the phase of the kicking field, or the timing of the kicking sequences (hence the evolution operator), is actively manipulated. In particular, we consider a MKR sys-

\footnotetext{
*Present address: Department of Chemistry and The James Franck Institute, University of Chicago, Chicago, IL 60637.
}

tem in which the sign of the kicking potential is periodically changed, or alternatively time delayed, after a certain number of kicks. As shown below, such a slight modification of the KR has profound effects on the dynamics: whereas periodically changing the sign of the kicking potential does not destroy DL, it dramatically changes the quantum diffusion dynamics of the KR as well as the nature of DL, offering opportunities for controlling the dynamics, as well as understanding quantum fluctuations and correlations in quantum chaos in periodically kicked systems. For example, we demonstrate that, with the sign of the kicking potential of the KR periodically changed, (i) the dynamical localization length is significantly increased so that the energy absorption is strongly enhanced; (ii) classical anomalous diffusion (which can be slower than quantum anomalous diffusion under certain conditions [20]) can enhance control even when the effective Planck constant is about an order of magnitude larger than the relevant classical phase space structures; and (iii) DL may display strong deviations from purely exponential line shapes.

This paper is organized as follows. In Sec. II we introduce the modified kicked-rotor model. We then present results in Sec. III on the enhancement of dynamical localization length, with qualitative explanations based upon a known result from the band random matrix theory [21-23]. In Sec. IV we show control of DL in a different regime, where the dynamics can be tied to a different mechanism, i.e., the creation of additional structures in classical phase space. Section $\mathrm{V}$ contains the results on coherent manipulations of the line shapes for DL. We conclude the paper with a brief discussion in Sec. VI.

\section{A MODIFIED KICKED-ROTOR MODEL}

The KR Hamiltonian is given by

$$
H^{K R}(\hat{L}, \theta, t)=\hat{L}^{2} / 2 I+\lambda \cos (\theta) \sum_{n} \delta(t / T-n),
$$

where $\hat{L}$ is the angular momentum operator, $\theta$ is the conjugate angle, $I$ is the moment of inertia, $\lambda$ is the strength of the kicking field, and $T$ is the time interval between kicks. The basis states of their Hilbert spaces are given by $|m\rangle$, with 
$\hat{L}|m\rangle=m \hbar|m\rangle$. The quantum KR map operator for propagating from time $\left(N-0^{+}\right) T$ to time $\left(N+1-0^{+}\right) T$ is given by

$$
\hat{F}_{K R}=\exp \left[i \frac{\tau}{2} \frac{\partial^{2}}{\partial \theta^{2}}\right] \exp [-i k \cos (\theta)],
$$

with dimensionless parameters $k=\lambda T / \hbar$ and the effective Planck constant $\tau=\hbar T / I$. For later use we also define the dimensionless scaled rotational energy as $\widetilde{E} \equiv\left\langle\hat{L}^{2}\right\rangle \tau^{2} / 2 \hbar^{2}$, where $\langle\cdot\rangle$ represents the average over the quantum ensemble. In the $|m\rangle$ representation, $\hat{F}_{K R}$ takes the following form [23]:

$$
\left\langle m_{1}\left|\hat{F}_{K R}\right| m_{2}\right\rangle=\exp \left(i \frac{\tau}{2} m_{1}^{2}\right) i^{m_{1}-m_{2}} J_{m_{1}-m_{2}}(k),
$$

where $J_{m_{1}-m_{2}}(k)$ is the Bessel function of the first kind of order $\left(m_{1}-m_{2}\right)$.

The classical limit of the KR quantum map, i.e., the standard map, depends on only one parameter $\kappa \equiv k \tau$ and is given by

$$
\begin{gathered}
\widetilde{L}_{N}=\widetilde{L}_{N-1}+\kappa \sin \left(\theta_{N-1}\right), \\
\theta_{N}=\theta_{N-1}+\widetilde{L}_{N},
\end{gathered}
$$

where $\widetilde{L} \equiv L \tau / \hbar$ is the scaled $c$-number angular momentum and $\left(\widetilde{L}_{N}, \theta_{N}\right)$ represents the phase space location of a classical trajectory at $\left(N+1-0^{+}\right) T$. For later discussion we note that for particular values of $\kappa$ the classical map Eq. (4) can generate accelerating trajectories whose momentum increases (or decreases) linearly with time (at least on the average). These trajectories are called transporting trajectories [24]. To see this, consider the initial conditions $\left(\widetilde{L}=2 \pi l_{1}\right.$, $\theta= \pm \pi / 2$ ) for $\kappa=2 \pi l_{2}$, where $l_{1}$ and $l_{2}$ are integers. Clearly, these phase space points are shifted by a constant value $\left( \pm 2 \pi l_{2}\right)$ in $\widetilde{L}$ after each iteration, resulting in a quadratic increase of rotational energy. These transporting trajectories are rather stable insofar as they may persist for values of $\kappa$ close to $2 \pi l_{2}$ (with their average momentum shift after each iteration oscillating around the constant value $\pm 2 \pi l_{2}$ ), thus giving rise to transporting regular islands [24], i.e., the accelerator modes in the KR case. If classical trajectories are launched from the accelerator modes, they simply jump to other similar islands located in adjacent phase space cells. For trajectories initially outside the accelerator modes, the "stickiness" of the boundary between the accelerator modes and the chaotic sea induces anomalous diffusion over the energy space, i.e., energy increases in a nonlinear fashion, but not quadratically. This is intrinsically different from the case of normal chaotic diffusion in which energy increases linearly with the number of kicks.

We introduce here a slightly modified kicked-rotor system whose Hamiltonian is given by

$$
H^{M K R}(\hat{L}, \theta, t)=\hat{L}^{2} / 2 I+\lambda \cos (\theta) \sum_{n} f_{M}(n) \delta(t / T-n),
$$

where $f_{M}(n)$ is real, $\left|f_{M}(n)\right|=1$, and $f_{M}(n)$ changes sign after every $M$ kicks. That is, the only difference between the KR and the MKR is that in the MKR the sign of the kicking potential is changed after every $M$ kicks. The effect of changing the sign of the kicking potential can be further understood in terms of the time-evolving wave function, which can be expanded as a superposition of $|m\rangle$ states: $\Sigma_{m} C_{m}\langle\theta \mid m\rangle$, with the expansion coefficients $C_{m}$. Since $\cos (\theta+\pi)=-\cos (\theta)$, and $\Sigma C_{m}\langle\theta+\pi \mid m\rangle$ $=\Sigma_{m}(-1)^{m} C_{m}\langle\theta \mid m\rangle$, changing the sign of the kicking potential is seen to be equivalent to adding a $\pi$ phase difference between all neighboring basis states. Compare this now to the effect of a time delay $t_{d}=2 \pi T / \tau$ between two neighboring kicks. Due to the free evolution of the rotor any two angular momentum eigenstates $|m\rangle$ and $|m+1\rangle$ will acquire in time $t_{d}$ an additional relative quantum phase given by $\exp \left\{i \pi t_{d}\left[(m+1)^{2}-m^{2}\right] /(2 T)\right\}=\exp [i(2 m+1) \pi]=\exp (i \pi) . \quad$ As such, the MKR can also be realized by introducing the time delay $t_{d}$ after every $M$ kicks.

For times $\left(N-0^{+}\right) T$ to $\left(N+M-0^{+}\right) T$, the MKR quantum propagator can be written as

$$
\hat{F}_{M K R}=\exp \left(i \pi \frac{\partial^{2}}{\partial \theta^{2}}\right) \hat{F}_{K R}^{M} \equiv \hat{D} \hat{F}_{K R}^{M}
$$

where this equation defines $\hat{D}$ as the free evolution operator over time $t_{d}$. Here $\hat{F}_{K R}^{M}$ denotes $M$ applications of $\hat{F}_{K R}$. From Eq. (6), one sees that the only difference in time propagation between the KR and the MKR for every $M$ kicks is the $\hat{D}$ operator, whose matrix elements $\left\langle m_{1}|\hat{D}| m_{2}\right\rangle$ are given by

$$
\left\langle m_{1}|\hat{D}| m_{2}\right\rangle=(-1)^{m_{1}} \delta_{m_{1} m_{2}} .
$$

Thus we have

$$
\left\langle m_{1}\left|\hat{F}_{M K R}\right| m_{2}\right\rangle=(-1)^{m_{1}}\left\langle m_{1}\left|\hat{F}_{K R}^{M}\right| m_{2}\right\rangle .
$$

Note also that the classical limit of the MKR quantum map [Eq. (6)] is given by

$$
\begin{gathered}
\widetilde{L}_{N+1}=\widetilde{L}_{N}+\kappa f_{M}(N) \sin \left(\theta_{N}\right), \\
\theta_{N}=\theta_{N}+\widetilde{L}_{N+1} .
\end{gathered}
$$

With respect to model systems in the literature, the MKR here can be regarded as a specific realization of the so-called generalized kicked-rotor model, which was first introduced in Ref. [25], in the context of quantum antiresonance. However, it is very different from the amplitude-modulated kicked rotor systems $[12,26]$ previously studied because (i) the kicking field strength here remains constant, and therefore any interesting results arise from pure phase modulations; and (ii) as shown below, the dynamical localization is significantly altered, but not destroyed. 

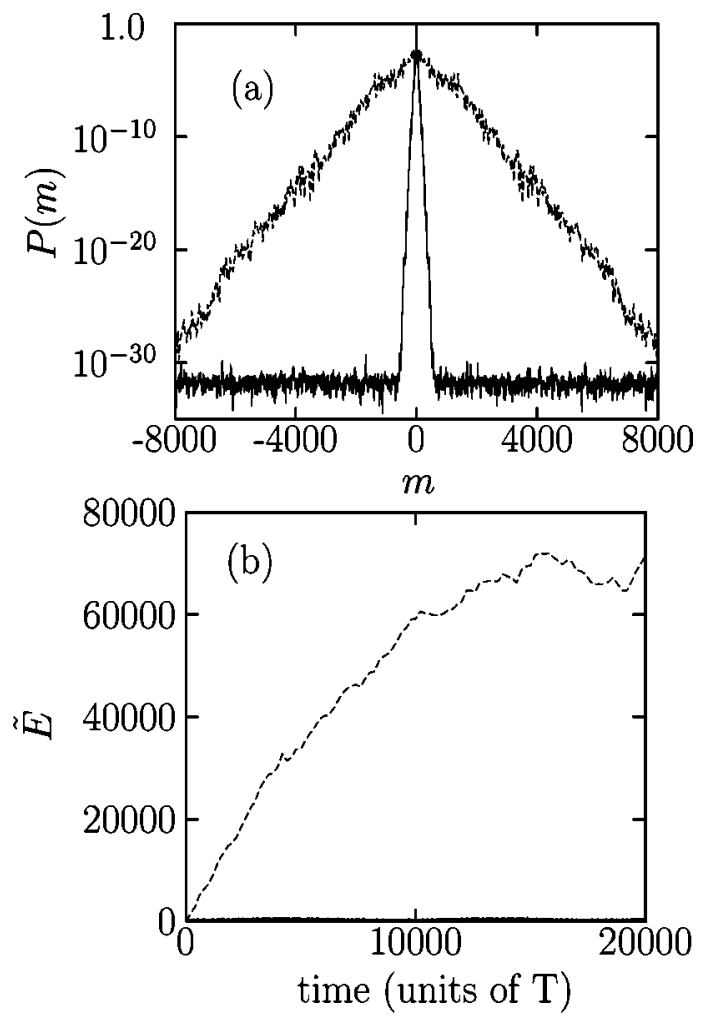

FIG. 1. Phase control of dynamical localization achieved by changing the sign of the kicking potential after every 50 kicks. $\kappa=4.0, \tau=2.0$, and the initial state is $|0\rangle$. (a) A comparison between the KR (the narrow line shape, solid line) and the MKR (the broad line shape, dashed line) in terms of the probability $P(m)$ of finding the system in the state $|m\rangle$ after $4 \times 10^{5}$ kicks. (b) The time dependence of the dimensionless scaled rotational energy $\widetilde{E}$ in each case of the KR (solid line) and the MKR (dashed line). Note that the solid line lies very close to the $\widetilde{E}=0$ axis.

\section{ENHANCED DYNAMICAL LOCALIZATION LENGTH}

As an example, consider the case of $k=4.0, \tau=2.0$, and $M=50$, whose classical limit for both the KR and the MKR is fully chaotic and displays normal chaotic diffusion. We demonstrate below that the dynamical localization and the related energy absorption associated with $\hat{F}_{M K R}$ are dramatically enhanced over that associated with $\hat{F}_{K R}^{M}$.

Figure 1(a) displays the angular momentum distribution $P(m)$ after $4 \times 10^{5}$ kicks, starting with the initial state $|0\rangle$, for both the KR and the MKR with $M=50$. The exponential line shape of $P(m)$ shown in Fig. 1(a) indicates that DL occurs in both cases. By fitting $P(m)$ with exponentials $P(m)$ $\sim \exp \left[-|m| / l_{K R}\right]$ and $P(m) \sim \exp \left[-|m| / l_{M K R}\right]$ for the $\mathrm{KR}$ and MKR, respectively, one obtains that the dynamical localization length $l_{M K R} \sim 140.0$ is significantly larger than $l_{K R}$ $\sim 7.0$. This clear difference in dynamical localization length is also reflected in the energy absorption shown in Fig. 1(b). In particular, while the energy absorption of KR saturates after a few kicks, the MKR system continues to absorb energy in a more or less linear manner for as long as $10^{4}$ kicks. Enhancement of dynamical localization length and energy absorption is also observed for other values of $M$, and for a wide range of parameters $k$ and $\tau$.

As is well known, the DL of the KR can be traced back to the localization properties of the eigenstates of the quantum map operator [Eq. (2)]. Note first that, due to the rapid decay of $J_{m_{1}-m_{2}}(k)$ with increasing $\left|m_{1}-m_{2}\right|$ and the pseudorandom nature of the function $\exp \left(i \pi m_{1}^{2} / 2\right)$ in $m_{1}$, the quantum map operator $\hat{F}_{K R}$ in general assumes a band structure and behaves in a pseudorandom manner in the $|m\rangle$ representation. Hence, below we qualitatively consider the MKR results in terms of a well-known feature from band random matrix theory [21-23], namely, that the larger the bandwidth of the quantum map operator the larger the dynamical localization length.

Note first that the matrix $\left\langle m\left|\hat{F}_{K R}\right| m^{\prime}\right\rangle$ is pseudorandom. However, we do not expect $\left\langle m\left|\hat{F}_{K R}^{M}\right| m^{\prime}\right\rangle$ to be a pseudorandom matrix of the same type since multiplying $\hat{F}_{K R}$ by itself $M$ times is expected to establish correlations between the matrix elements $\left\langle m_{1}\left|\hat{F}_{K R}^{M}\right| m_{2}\right\rangle$. Nevertheless, we do assume that the matrix $\left\langle m\left|\hat{F}_{M K R}\right| m^{\prime}\right\rangle$ is banded and pseudorandom since the eigenstates of $\hat{F}_{M K R}$ have no simple connection with those of $\hat{F}_{K R}$. Consider now an arbitrary matrix element $\left\langle m_{1}\left|\hat{F}_{K R}^{M}\right| m_{2}\right\rangle$. Due to the quantum diffusive dynamics within the $M$ kicks, the $\left\langle m_{1}\left|\hat{F}_{K R}^{M}\right| m_{2}\right\rangle$ with $\left|m_{1}-m_{2}\right| \gg 1$ should be much greater than $\left\langle m_{1}\left|\hat{F}_{K R}\right| m_{2}\right\rangle$ (nevertheless, both of them can be very small). According to Eq. (8), this implies that the matrix element $\left\langle m_{1}\left|\hat{F}_{M K R}\right| m_{2}\right\rangle$ is also much greater than $\left\langle m_{1}\left|\hat{F}_{K R}\right| m_{2}\right\rangle$. In this sense, we expect that the $\left\langle m\left|\hat{F}_{M K R}\right| m^{\prime}\right\rangle$ matrix should display a wider band than does $\left\langle m\left|\hat{F}_{K R}\right| m^{\prime}\right\rangle$.

The bandwidth of the matrix $\left\langle m\left|\hat{F}_{K R}\right| m^{\prime}\right\rangle$ can be defined by choosing a cutoff value for its matrix elements. One traditional choice is $\left|m_{1}-m_{2}\right| \sim k$. In this case, $\left\langle m_{1}\left|\hat{F}_{K R}\right| m_{2}\right\rangle$ at the boundary of the band is on the order of $J_{k}(k)$, a number which is sufficiently small. However, this cutoff value, if applied to the matrix $\left\langle m\left|\hat{F}_{M K R}\right| m^{\prime}\right\rangle$, would yield almost the same bandwidth as $\hat{F}_{K R}$. Hence, quantitatively characterizing the band structure of the matrix $\left\langle m\left|\hat{F}_{M K R}\right| m^{\prime}\right\rangle$ is subtle, since the very small matrix elements $\left\langle m_{1}\left|\hat{F}_{M K R}\right| m_{2}\right\rangle$ must play an important role in enhancing the dynamical localization length of the MKR.

To gain more insight we computationally examined the $M$ dependence of the dynamical localization length of the MKR. Specifically, we numerically diagonalized $\hat{F}_{M K R}$, where each matrix is generated using $2^{14}$ basis states and is then truncated at dimension $d$ chosen below. We characterize the average dynamical localization length by the Shannon entropy $S_{M K R}$ [27] (note that the Shannon entropy is simply proportional to the dynamical localization length [27]) averaged over all approximate eigenstates $\left|\phi_{j}\right\rangle$ of $\hat{F}_{M K R}$, i.e.,

$$
S_{M K R}=\frac{2}{\alpha d} \sum_{j=1}^{d} \exp \left[-\sum_{m=-d / 2}^{d / 2}\left|\left\langle m \mid \phi_{j}\right\rangle\right|^{2} \ln \left|\left\langle m \mid \phi_{j}\right\rangle\right|^{2}\right],
$$



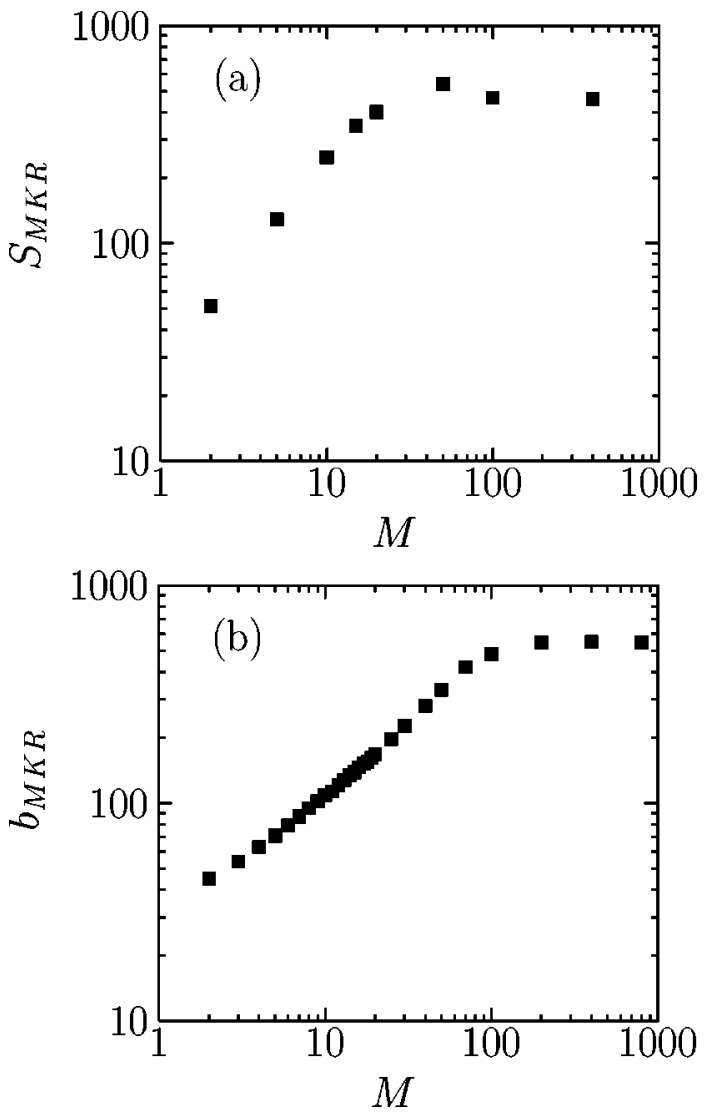

FIG. 2. (a) The $M$ dependence of the dynamical localization length of the MKR, characterized by the Shannon entropy $S_{M K R}$ averaged over all approximate eigenfunctions of the MKR propagator $\hat{F}_{M K R}$. (b) The $M$ dependence of the bandwidth $b_{M K R}$ associated with the MKR propagator $\hat{F}_{M K R}$, defined by a cutoff value (as small as $10^{-20}$ ) for its matrix elements $\left\langle m_{1}\left|\hat{F}_{M K R}\right| m_{2}\right\rangle$.

where the constant $\alpha$ equals 0.96 , and $d$ is chosen to be 2700 . The results for the $M$ dependence of $S_{M K R}$ for $M=2$ to $M$ $=400$ are shown in a log-log plot in Fig. 2(a). $S_{M K R}$ is seen to behave initially as a smoothly increasing function of $M$, and then to saturate at $M \sim 50$. To explain the results from the perspective of band random matrix theory, we choose a cutoff value for matrix elements $\left\langle m_{1}\left|\hat{F}_{M K R}\right| m_{2}\right\rangle$ so as to define the bandwidth $b_{M K R}$. Interestingly, we find that this cutoff value must be extremely small (roughly speaking, at least as small as $10^{-10}$ ) in order that the $M$ dependence of $b_{M K R}$ resembles that of $S_{M K R}$. For example, Fig. 2(b) displays $b_{M K R}$ as a function of $M$ for a cutoff value of $10^{-20}$. The evident similarities between Fig. 2(a) and Fig. 2(b) suggest (i) that we can indeed qualitatively explain the enhanced dynamical localization length in terms of the band random matrix theory, and (ii) that even extremely small quantum fluctuations in the values of the matrix elements of the MKR quantum map operator affect its dynamical localization length.

In particular, comparing Fig. 2(a) with Fig. 2(b), one sees that the saturation behavior of $S_{M K R}$ for large $M$ is consistent with the saturation behavior of $b_{M K R}$. The latter reflects the saturation of the matrix elements $\left\langle m_{1}\left|\hat{F}_{M K R}\right| m_{2}\right\rangle$ and there- fore the matrix elements $\left\langle m_{1}\left|\hat{F}_{K R}^{M}\right| m_{2}\right\rangle$. As such, we infer that the saturation behavior of $S_{M K R}$ is simply a result of DL in the KR.

Detailed studies on numerous other cases with varying $k$ and $\tau$ show that the above result, i.e., $S_{M K R}$ first increases with $M$ and then saturates, is quite general, as long as the system has a completely chaotic classical limit and quantum correlations are insignificant. On the other hand, if $S_{M K R}$ behaves differently, then there are two possible origins: either the system is in the deep quantum regime or there are non-negligible regular islands in the classical phase space. For example, in the next section we show cases in which the energy absorption in the MKR with $M=2$ is appreciably larger than that in the MKR with $M=3$. In these cases one can obtain even more significant changes in DL.

\section{CLASSICAL ANOMALOUS DIFFUSION ASSISTED CONTROL}

In the previous section we studied cases where both the KR and the MKR essentially have a fully chaotic classical limit. However, as shown below, the MKR can also display nonchaotic classical phase space structures that are absent in the KR. In particular, regular islands with very interesting transporting properties can be induced in the MKR. In such cases one can achieve even more dramatic alteration of the DL than that shown above.

Consider first the classical MKR map Eq. (9) for $M=2$. Interestingly, in this case there exist transporting trajectories that are different from those in the KR. In particular, we have previously observed [20] that for $\kappa=\left(2 l_{2}+1\right) \pi$ trajectories emanating from $\widetilde{L}=\left(2 l_{1}+1\right) \pi, \theta= \pm \pi / 2$ will be shifted by a constant value $\left[ \pm\left(2 l_{2}+1\right) \pi\right]$ in $\widetilde{L}$ after each kick. This observation suggests that transporting regular islands that differ from the accelerator modes in the KR can be created by changing the sign of the kicking potential after every two kicks. This is confirmed in our extensive numerical studies, both here and in Ref. [20].

Note that in our previous work [20] we were most interested in the quantum-classical comparison in anomalous diffusion and considered relatively small effective Planck constants. In that case we found that quantum anomalous diffusion induced by the transporting regular islands can be much faster than the underlying classical anomalous diffusion. Here, to make a closer connection to atom optics experiments, we consider larger $\tau \sim 1.0$. In these cases the effective Planck constant is about an order of magnitude larger than the area of the phase space structures associated with classical anomalous diffusion. Intuitively, one would anticipate that such transporting regular islands are too small to be relevant to the quantum dynamics. Surprisingly, this intuition is incorrect, as shown below.

To be more specific, consider first the case of $\kappa=5.0$. Figure 3 displays the classical phase space structures of both the KR and the MKR with $M=2$. While the regular islands seen in Fig. 3(a) (the KR case) are not transporting (that is, the momentum of the trajectories launched from these islands is bounded and oscillates periodically), a simple com- 
(a)

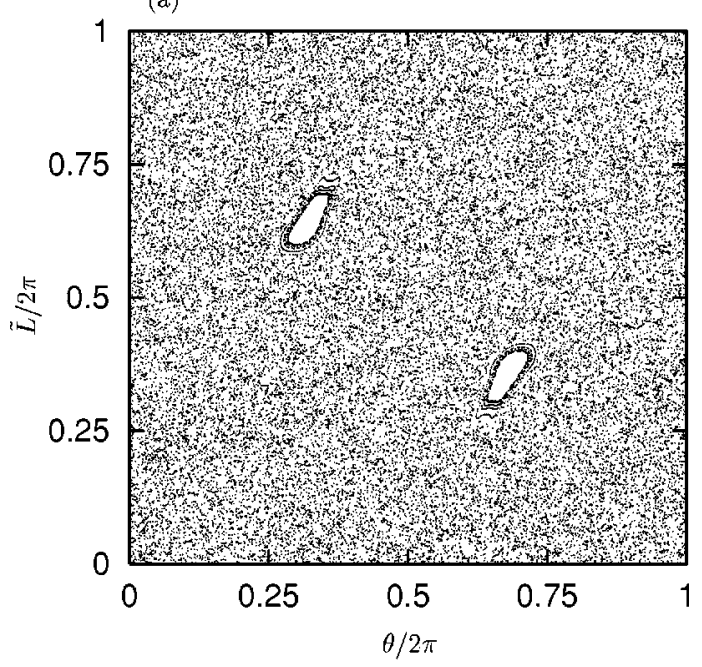

(b)

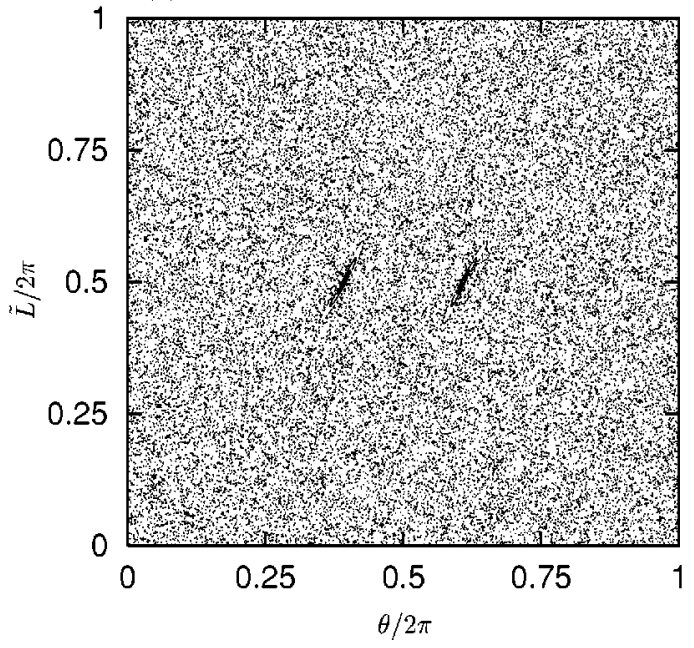

FIG. 3. Classical phase space structures of (a) the standard map and (b) the map of Eq. (9) with $M=2$, in the case of $\kappa=5.0$. All variables are in dimensionless units. Note that the small regular islands seen in panel (b) are transporting while those in panel (a) are not.

putation reveals that the small islands seen in Fig. 3(b) in the MKR case are transporting. That is, classical trajectories launched from the right (left) transporting regular island shown in Fig. 3(b) have their momentum shifted by $\pi(-\pi)$ on the average after each kick, indicating that these islands originate from the marginally stable point $\widetilde{L}=\left(2 l_{1}+1\right) \pi$, $\theta= \pm \pi / 2$ with $\kappa=\pi$. Hence, in this case phase manipulation in going from the KR to the MKR has both destroyed the nontransporting regular islands of KR and induced transporting regular islands. Consider a second case with $\kappa=10.0$. The corresponding classical phase space structures are shown in Fig. 4(a) (KR) and Fig. 4(b) (MKR) (Note that, to clearly display the transporting regular islands, only a part of one phase space cell is shown here.) While there are hardly any regular islands seen in Fig. 4(a), two small transporting regular islands are seen in Fig. 4(b). The average momentum shift for each kick associated with these two islands is found to be $\pm 3 \pi$, consistent with the fact that $\kappa=10.0$ is close to $3 \pi$.
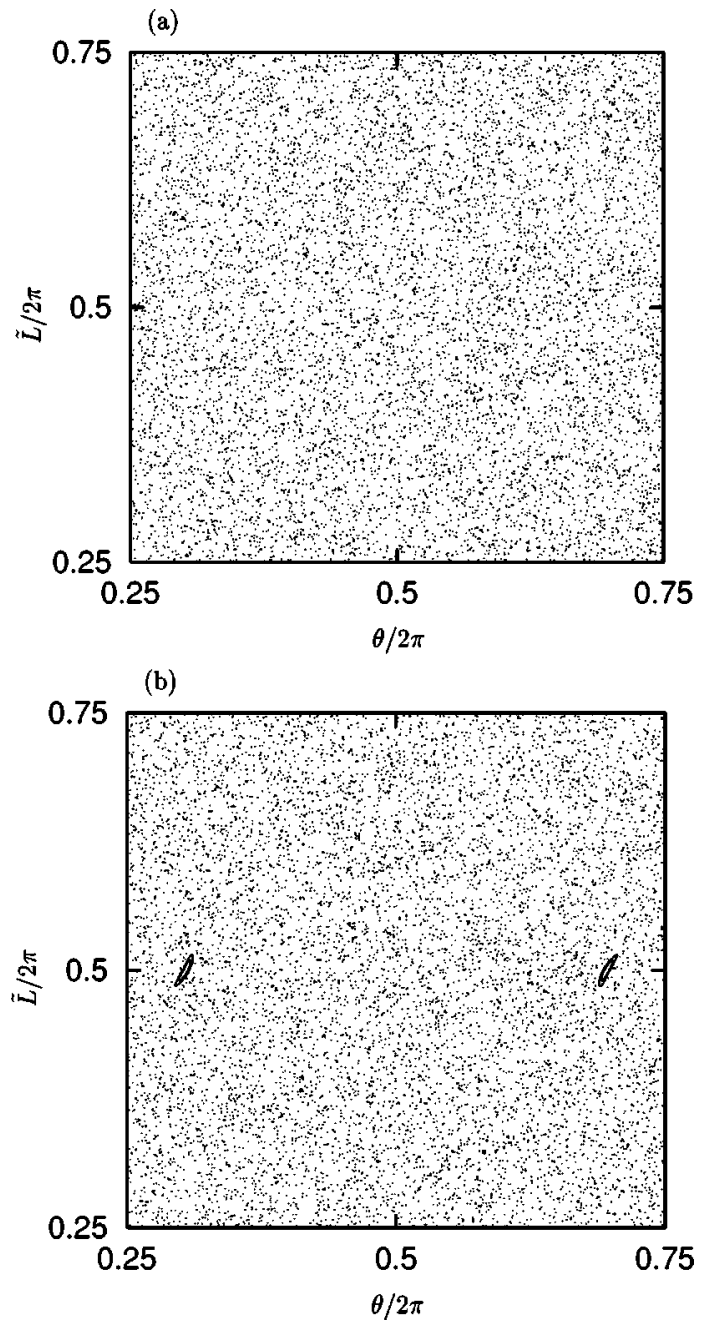

FIG. 4. As in Fig. 3 except $\kappa=10.0$. Note that the small regular islands seen in panel (b) are transporting.

Consider now the quantum dynamics of these systems. There have been only a few studies on the quantum dynamics of $\delta$-function-kicked systems where classical chaos coexists with transporting regular islands. Of particular relevance is the previous result that the accelerator modes of the KR enhance deviations from the normal DL behavior in the KR $[28,29]$, even for systems far from the semiclassical limit. Since the MKR displays additional transporting islands, we therefore anticipate that DL may be strongly affected by modifying the Hamiltonian from the KR to the MKR system. This is indeed seen below. The results are, however, counterintuitive, since the classical transporting regular islands created by phase manipulation of the kicking field are found to have an area that is much smaller than the effective Planck constant.

For example, for each of the KR and MKR, Figs. 5 and 6 display energy absorption for the cases of $\tau=1.0, k=5.0$ and $\tau=1.0, k=10.0$ (corresponding to $\kappa=5$ and $\kappa=10$ ), respectively. Also shown is the MKR case with $M=3$, discussed below. It is seen that the energy absorption associated with the MKR with $M=2$ (upper dashed line) is much larger than that of the KR (solid line). Consider, for example, $\widetilde{E}$ at a 


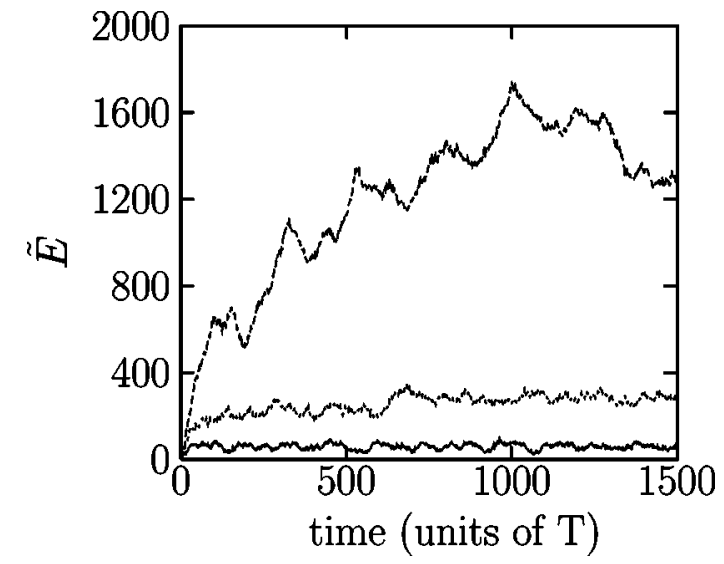

FIG. 5. The time dependence of the dimensionless scaled rotational energy $\widetilde{E}$ for the KR (solid line), for the MKR with $M=2$ (uppermost dashed line), and for the MKR with $M=3$ (middle dashed curve), with $\tau=1.0, k=5.0$, and the initial state $|0\rangle$.

specific time $t=1500 T$ when the energy absorption of both the KR and the MKR has clearly shown signs of saturation (e.g., the average rotational energy may decrease with time due to statistical fluctuations). In the first case (Fig. 5), $\widetilde{E}$ $=67.7$ for the KR and $\widetilde{E}=1269.7$ for the MKR. In the second case (Fig. 6), $\widetilde{E}=798.0$ for the KR and $\widetilde{E}=10049.0$ for the MKR. In both cases a control factor larger than an order of magnitude has been achieved in going from the KR Hamiltonian to the MKR case with $M=2$.

This is not the case for $M=3, \tau=1.0, k=5.0$ shown in Fig. 5. Here the energy absorption in the MKR with $M=3$ is only slightly larger than in the KR and far less than in the MKR with $M=2$. Similarly, for the case of $\tau=1.0, k$ $=10.0$ shown in Fig. 6, although energy absorption in the MKR with $M=3$ is much enhanced (compared with the $\mathrm{KR}$ ), it is still not as significant as in the MKR with $M$ $=2$. This indicates, as confirmed by directly examining $P(m)$ (not shown) after saturation, that for both cases the dynamical localization length of the MKR with $M=3$ is no larger than that of the MKR with $M=2$, contrary to what is observed in the previous section. This is because the underlying classical dynamics of MKR here is not completely cha-

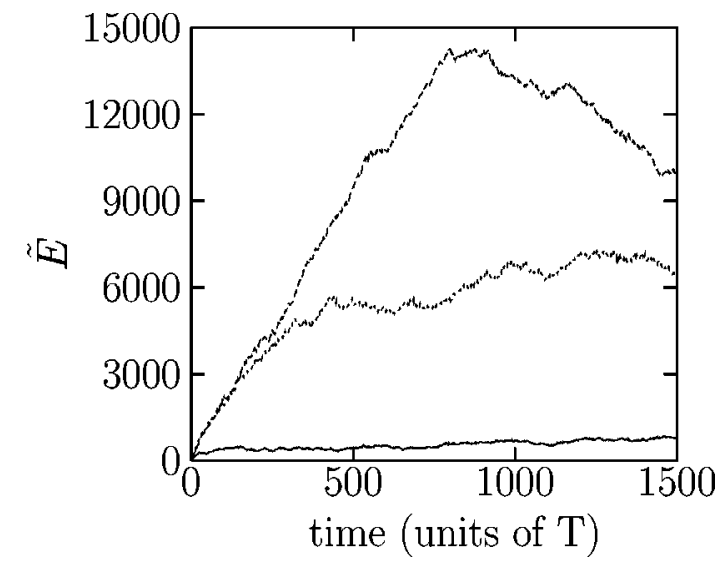

FIG. 6. As in Fig. 5 except $\tau=1.0, k=10.0$. otic, i.e., the classical dynamics displays characteristics of anomalous diffusion due to transporting regular islands in phase space. Thus one can expect statistical deviations from the band random matrix theory, used previously to relate $M$ to the extent of control.

These results emphasize that the control mechanism here is uniquely based upon the transporting regular islands created by our control scenario. This is further supported by the line shape for DL, which can be strongly nonexponential, as discussed below.

\section{NONEXPONENTIAL LINE SHAPES FOR DYNAMICAL LOCALIZATION}

It was pointed out more than two decades ago [5] that the DL of the KR can be mapped onto the problem of Anderson localization in disordered systems. In particular, an exactly soluble case of disorder in tight-binding models, i.e., the Lloyd model [30], suggests that dynamical localization should assume an exponential line shape, at least on the average. This has been confirmed by numerous computational studies on the KR. For example, Fig. 1(a) clearly demonstrates, for both the KR and the MKR, that the distribution function $P(m)$ can be fitted beautifully with an exponential function with a characteristic localization length.

However, the Hamiltonian nature of the KR and MKR implies that there always exist some subtle quantum phase correlations in the quantum dynamics. Hence, in addition to some universal properties of DL, the DL line shape can display rich nonuniversal properties, e.g., the system may display nonexponential dynamical localization. Nonexponential dynamical localization has been previously observed in the KR but its origins are still poorly understood [28,31].

Here, we demonstrate that the MKR with $M=2$ can display strongly nonexponential line shapes for DL, rarely seen in the KR. We focus on the MKR with $M=2$ since the classical MKR with $M=2$ has transporting regular islands that are absent in the KR. Second, transporting regular islands may induce large fluctuations in DL [28]. However, we examine below cases with connections to anomalous diffusion as well as those without clear connections to anomalous diffusion. We have also studied other versions of the MKR with $M \neq 2$, and have found that nonexponential line shapes for DL in the latter case are much less common than in the $M$ $=2$ case.

Figure 7 compares nonexponential line shapes for DL in the MKR (upper dashed lines) to the analogous exponential line shapes for DL in the KR (solid lines), for four different values of $k$ and $\tau$. The line shapes are obtained by propagating the quantum dynamics for 8000 kicks from the initial state $|0\rangle$, and will remain essentially the same for longer propagation times [32]. The huge difference between the KR and MKR line shapes is striking. As shown in Fig. 7, in the MKR case, $P(m)$ plotted on a logarithmic scale displays structures that are far from a purely exponential line shape. For example, one sees that the initial exponential decay rate of $P(m)$ with $|m|$ is considerably smaller than its large- $|m|$ exponential decay rate, suggesting that multiple characteristic lengths are needed to describe the DL of the MKR. It is 

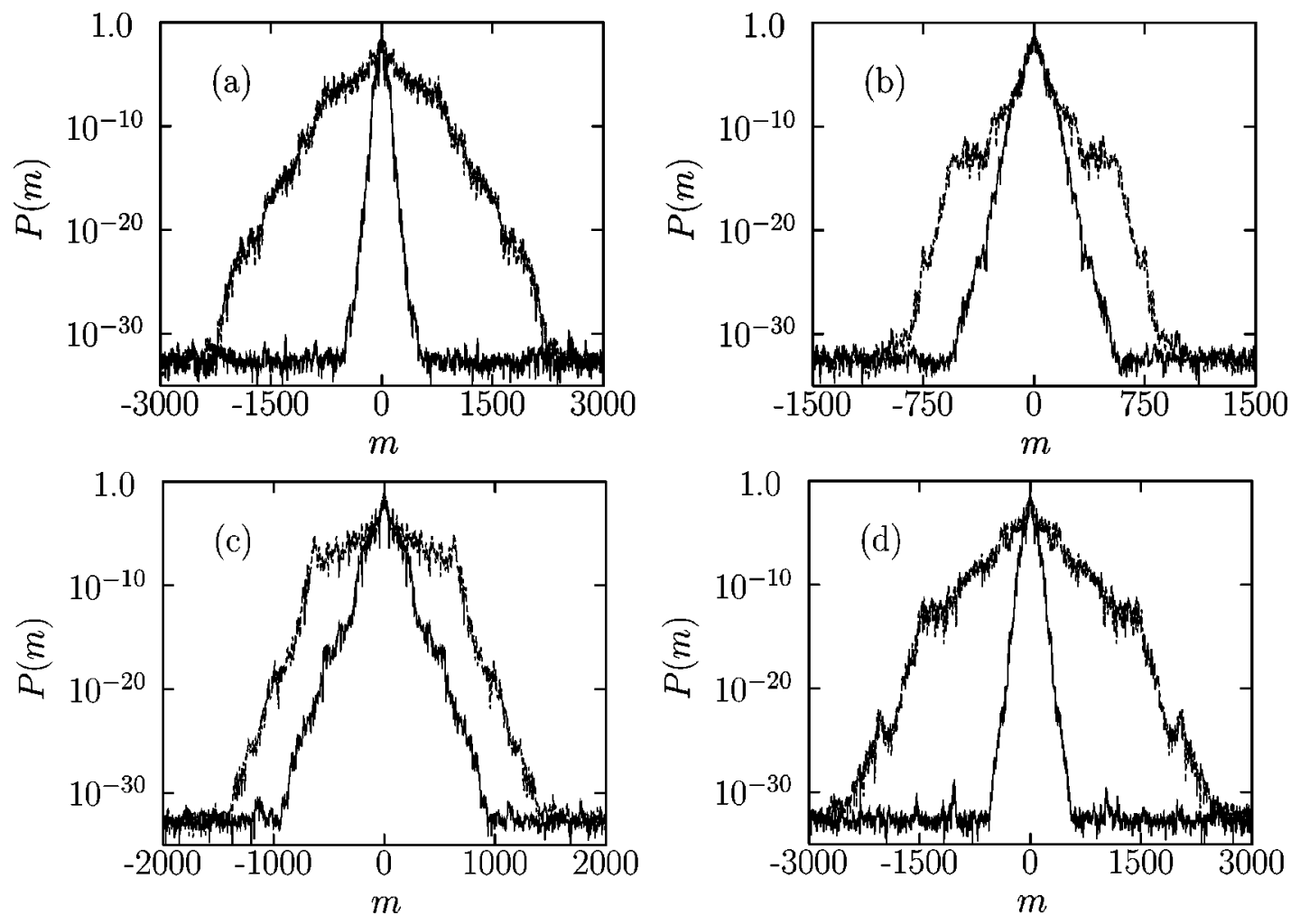

FIG. 7. Four examples of nonexponential line shapes for dynamical localization in the MKR with $M=2$, shown in terms of the probability $P(m)$ of finding the system in the state $|m\rangle$ after 8000 kicks, with the initial state $|0\rangle$. In each case the broad line shape is associated with the MKR, and for the purpose of comparison, the narrow line shape of the analogous KR is also shown. (a) $\tau=1.0$, $k=5.0$, (b) $\tau=2.0, k=5.0$, (c) $\tau=1.0, k=5.7$, and (d) $\tau=2.0, k=6.0$.

also seen that the difference in $P(m)$ between KR and MKR can be as large as ten orders of magnitude or more. Further, Figs. 7(b) and 7(c) indicate that the decay rate of $P(m)$ for large $|m|$ in the MKR case is very similar to that in the KR case, although this is not the case in Figs. 7(a) and 7(d).

To the best of our knowledge, this is the first demonstration that intriguing differences in the line shapes for DL can be created by simply changing the sign of the kicking potential periodically.

The four cases of nonexponential line shapes shown in Fig. 7 can be divided into two categories, based upon the properties of their underlying classical dynamics. The classical dynamics associated with the cases in Figs. 7(a) and 7(b), shown in Figs. 3(b) and 4(b), displays transporting regular islands. The presence of these classical structures implies an inhomogeneous classical phase space, and, as demonstrated in the previous section, may have a significant impact on the quantum dynamics even when their size is much smaller than the effective Planck constant. In this regard, the nonexponential line shapes shown in Figs. 7(a) and 7(b) may not be totally surprising. However, for the other two MKR cases shown in Figs. 7(c) and 7(d), we did not find any regular islands in their classical phase space even when examined on a very fine scale, suggesting that their classical dynamics is essentially fully chaotic. Thus, understanding the nonexponential line shapes shown in Fig. 7(c) and Fig. 7(d) will be even more challenging.
The nonexponential line shapes for DL arise from extremely small quantum fluctuations and residual quantum correlations in quantum chaos. To be able to resolve the nonexponential line shape for DL, $P(m)$ has to be known with high precision. For example, the two shoulders shown in Fig. 7(b) involve occupation probabilities $P(m)$ as small as $\sim 10^{-14}$. It is therefore not surprising that, while it is common to have strongly nonexponential line shapes for DL in the MKR case, each individual line shape is highly sensitive to the exact value of the effective Planck constant. For example, for the case shown in Fig. 7(b), increasing the value of $\tau$ from 2.0 to $2.0+10^{-5}$ can completely destroy the nonexponential line shape. This drastic change in the line shape for DL even occurs without causing an obvious difference in energy absorption behavior. Evidently, then, both experimental observations and theoretical predictions of nonexponential dynamical localization are far from trivial and are in need of further study.

\section{DISCUSSION AND CONCLUSIONS}

This paper has dealt with control of dynamical localization in kicked-rotor systems. In all cases we manipulated the external kicking field to alter the properties of the rotor system, i.e., the distribution of population among rotor energy levels after saturation as well as the energy absorption. In particular, we have examined the effect of introducing a reversal of the kicking field after $M$ kicks which, within the 
framework of quantum mechanics, corresponds to introducing a phase shift among rotor energy levels.

Two parameter regimes have been examined, one which shows enhanced DL lengths with increasing $M$, and the other which need not. This behavioral difference can be understood in terms of the character of the underlying classical phase space: the former systems are completely chaotic whereas the latter show a mixed phase space that includes transporting regular islands. Indeed, we have found that even if the transporting islands are tiny compared to the effective Planck constant, they still have a profound effect on the control of the DL. Further, a comparison of traditional kickedrotor system to modified kicked-rotor systems shows that the latter are much more capable of displaying nonexponential dynamical localization. Thus, by modifying the kicking potential we are able to control the dynamical localization in the kicked rotor.

The results of this paper are relevant to two fields of study: quantum control and kicked-rotor dynamics. From the control perspective, modifying the kicking field changes the dynamics. However, this system does not obviously permit a picture in terms of interfering quantum pathways (the standard view of weak field coherent control $[15,16]$ ) since (a) the kicking field is always on, and (b) there are a multitude of interfering transitions responsible for the observed behavior. Indeed, it is even difficult to isolate the interfering pathways that are responsible for dynamical localization in the simple kicked rotor, whose dynamics is easier than that of the MKR.

From the viewpoint of kicked-rotor studies in the field of quantum chaos, this paper provides insights into the quantum dynamics in the case displaying classical anomalous diffusion. As one of the results of this study, we find that classical transporting regular islands can dramatically affect the quantum dynamics even when their size is much smaller than the effective Planck constant. Further, the MKR system proposed in this paper provides a model for the study of quantum dynamics where the underlying classical chaos coexists with transporting regular islands. By choosing proper system parameters, we can create transporting regular islands whose size varies from being much larger to being much smaller than that of the accelerator modes of KR. Encouraged by this, we plan in the near future to further use the MKR to study quantum tunneling between the transporting regular island and the chaotic sea [20,29] and between transporting regular islands, and to study the phase space structure of quantum eigenstates [24].

In the fully chaotic case, our approach suggests the need for additional studies of dynamical localization from the band random matrix theory perspective. For example, we have qualitatively explained the results in Sec. III in terms of well-known features of band random matrix theory. However, we found that the bandwidth of the quantum map operator had to be defined using an extremely small cutoff value for the matrix elements, suggesting that a quantitative understanding of MKR results such as Fig. 2 may require different models of band random matrix ensembles.

The strongly nonexponential line shapes for DL found in the MKR with $M=2$ further demonstrate the need for more theoretical work on properties of DL. In particular, our results should motivate greater interest in characterizing and understanding nonexponential dynamical localization, with efforts directed toward explaining why nonexponential dynamical localization occurs for some system parameters and not for others. This is of importance in understanding the high sensitivity of nonexponential line shapes for DL to the exact value of the effective Planck constant.

We have chosen the system parameters to be within the reach of current atom optics experiments on the KR [2]. Although experimental studies of nonexponential line shapes for DL are difficult, we believe that it is straightforward to experimentally observe the results of Sec. III and Sec. IV. Apart from the atom optics realization of KR and MKR, it is also interesting to consider a molecular version of KR and MKR, i.e., diatomics periodically kicked in strong microwave fields [5,18]. Preliminary computational studies [33] confirm that directly observing quantum control of dynamical localization in molecular rotational motion is possible, e.g., in the case of quantum anomalous diffusion. Another promising experimental realization of KR and MKR requires kicked particles in a square-well potential [34]. Along this direction an interesting model (which is very different from ours) has recently been proposed for the study of classical and quantum anomalous diffusion [35].

In summary, consideration of control in classically chaotic quantum systems is of general interest and importance to both the fields of quantum chaos and quantum control. In this paper we have demonstrated, via a modified kicked-rotor model, that dynamical localization, perhaps the best known phenomenon in quantum chaos, can be modified over a wide range. The results are of both experimental and theoretical interest.

\section{ACKNOWLEDGMENTS}

This work was supported by the U.S. Office of Naval Research and the Natural Sciences and Engineering Research Council of Canada. H.J.W. was partially supported by the Studien-stiftung des Deutschen Volkes and the Barth Fonds of ETH Zürich.
[1] G. Casati and B. Chirikov, Quantum Chaos: Between Order and Disorder (Cambridge University Press, New York, 1995).

[2] F.L. Moore et al., Phys. Rev. Lett. 75, 4598 (1995); H. Ammann et al., ibid. 80, 4111 (1998); J. Ringot et al., ibid. 85, 2741 (2000); M.B. d'Arcy et al., ibid. 87, 074102 (2001).

[3] S. Fishman, D.R. Grempel, and R.E. Prange, Phys. Rev. Lett. 49, 509 (1982).
[4] G. Benenti, G. Casati, I. Guarneri, and M. Terraneo, Phys. Rev. Lett. 87, 014101 (2001).

[5] R. Blümel, S. Fishman, and U. Smilansky, J. Chem. Phys. 84, 2604 (1986).

[6] I.Sh. Averbukh and R. Arvieu, Phys. Rev. Lett. 87, 163601 (2001).

[7] P. Facchi, S. Pascazio, and A. Scardicchio, Phys. Rev. Lett. 83, 
61 (1999).

[8] B. Georgeot and D.L. Shepelyansky, Phys. Rev. Lett. 86, 2890 (2001).

[9] E. Ott, T.M. Antonsen, Jr., and J.D. Hanson, Phys. Rev. Lett. 53, 2187 (1984).

[10] D. Cohen, Phys. Rev. A 44, 2292 (1991).

[11] R. Graham and S. Miyazaki, Phys. Rev. A 53, 2683 (1996); P. Goetsch and R. Graham, ibid. 54, 5345 (1996).

[12] G. Casati, I. Guarneri, M. Leschanz, D.L. Shepelyansky, and C. Sinha, Phys. Lett. A 154, 19 (1990).

[13] G. Abal, R. Donangelo, A. Romanelli, A.C. Sicardi Schifino, and R. Siri, Phys. Rev. E 65, 046236 (2002).

[14] S.A. Rice and M. Zhao, Optical Control of Molecular Dynamics (Wiley, New York, 2000).

[15] M. Shapiro and P. Brumer, Adv. At., Mol., Opt. Phys. 42, 287 (2000).

[16] M. Shapiro and P. Brumer, Principles of the Quantum Control of Molecular Processes (Wiley, New York, 2003).

[17] J. Gong and P. Brumer, Phys. Rev. Lett. 86, 1741 (2001).

[18] J. Gong and P. Brumer, J. Chem. Phys. 115, 3590 (2001).

[19] J. Gong and P. Brumer, Phys. Rev. Lett. 88, 203001 (2002).

[20] J. Gong, H.J. Wörner, and P. Brumer, Phys. Rev. E 68, 026209 (2003).

[21] G. Casati, L. Molinari, and F. Izrailev, Phys. Rev. Lett. 64, 1851 (1990).

[22] J. Gong and G. Xu, Commun. Theor. Phys. 31, 91 (1999);
W.G. Wang, Phys. Rev. E 65, 066207 (2002).

[23] F.M. Izrailev, Phys. Rep. 196, 299 (1990).

[24] See, for example, L. Hufnagel, R. Ketzmerick, M.F. Otto, and H. Schanz, Phys. Rev. Lett. 89, 154101 (2002).

[25] I. Dana, E. Eisenberg, and N. Shnerb, Phys. Rev. E 54, 5948 (1996).

[26] D.L. Shepelyansky, Physica D 8, 208 (1983).

[27] G. Casati, I. Guarneri, F. Izrailev, and R. Scharf, Phys. Rev. Lett. 64, 5 (1990).

[28] B. Sundaram and G.M. Zaslavsky, Phys. Rev. E 59, 7231 (1999).

[29] A. Iomin, S. Fishman, and G. Zaslavsky, Phys. Rev. E 65, 036215 (2002).

[30] P. Lloyd, J. Phys. C 2, 1717 (1972).

[31] I.I. Satija, B. Sundaram, and J.A. Ketoja, Phys. Rev. E 60, 453 (1999).

[32] We have also examined the eigenstates of the quantum MKR propagator and found that a significant number of eigenstates display nonexponential line shapes that are similar to what is seen from propagating the initial state $|0\rangle$.

[33] H.J. Wörner, J. Gong, and P. Brumer (unpublished).

[34] R. Sankaranarayanan, A. Lakshminarayan, and V.B. Sheorey, Phys. Rev. E 64, 046210 (2001).

[35] R. Sankaranarayanan, A. Lakshminarayan, and V.B. Sheorey, e-print nlin. CD/0212003. 